\title{
Sudakov suppression of the Balitsky-Kovchegov kernel
}

\author{
Du-xin Zheng and Jian Zhou \\ Institute of Frontier and Interdisciplinary Science, Shandong University, \\ Binghai Street, Qingdao, China \\ E-mail: 18264156329@163.com, jzhou@sdu.edu.cn
}

ABSTRACT: To sum high-energy leading logarithms in a consistent way, one has to impose the strong ordering in both projectile rapidity and dense target rapidity simultaneously, which results in a kinematically improved Balitsky-Kovchegov (BK) equation. We find that beyond this strong ordering region, the important sub-leading double logarithms arise at high order due to the incomplete cancellation between real corrections and virtual corrections in a t-channel calculation. Based on this observation, we further argue that these double logarithms are the Sudakov type ones, and thus can be resummed into an exponential leading to a Sudakov suppressed BK equation.

KEYwORDS: Perturbative QCD, Resummation

ARXIV EPRINT: 1906.06825 


\section{Contents}

\section{Introduction 1}

2 Derivation of the double logarithm correction to the BK kernel at two loop order and beyond 3

2.1 Non-local BK equation and kinematical constraints 5

2.2 The complete and incomplete cancellations between real graphs and virtual $\begin{array}{ll}\text { graphs } & 7\end{array}$

$\begin{array}{lll}2.3 & \text { Multiple soft gluon radiations } & 11\end{array}$

$\begin{array}{lll}3 & \text { Summary } & 13\end{array}$

\section{Introduction}

The non-linear evolution equation — the Balitsky-JIMWLK equation [1-4] or its meanfield truncation: the BK equation $[1,5]$ plays a central role in studying saturation physics. The leading order evolution kernels have been derived about two decades ago. To make realistic predictions for observables in various high energy scattering processes at RHIC, LHC and the future EIC, it is necessary to utilize the NLO version of the BK equation in phenomenology studies. Though the calculation of NLO BK kernel is extremely complicated, it has been eventually achieved in ref. [6]. Later, there was also attempt to extend the NLO analysis to the Balitsky-JIMWLK equation [7, 8].

However, when trying to solve the NLO BK equation numerically [9], it was found to be unstable. This is essentially due to the NLO correction enhanced by double logarithms, which becomes very large when the parent dipole is much smaller than the daughter dipole. As a matter of fact, the similar problem was earlier observed in the context of the BFKL dynamics $[10,11]$ as well. To cure the instability issue and improve the convergence of the perturbation series, significant efforts were then devoted to resum these large double logarithms to all orders in the dilute limit [12-16] and in the saturation case [18-22]. The common feature of various proposed resummation schemes is to implement a kinematical constraint for successive gluon radiations during small $x$ evolution. It has been argued that these large double logarithms can be effectively resummed to all orders via introducing a kinematically improved BK equation.

It is indeed a natural idea to impose a kinematical constraint in the evolution kernels as the derivations of the BK/BFKL equations rely on making systematical kinematical approximations in strong rapidity ordering region. In particular, to construct a self-consistent small $x$ evolution, the strong ordering in both projectile rapidity and dense target rapidity must be satisfied simultaneously. The BK equation is conventionally formulated as an evolution with the projectile rapidity. The strong ordering in projectile rapidity is realized by 
construction in this case, whereas the strong target rapidity ordering is not automatically guaranteed. One way of enforcing the target rapidity ordering is to insert a theta function in the LO BK kernel. The most important part of the double logarithms arises at high order corrections is shown to be recovered from a such non-local BK equation [18]. Alternatively, the double-logarithmic corrections can be explicitly resummed in the kernel and give rise to a collinearly-improved BK equation $[19,20]$. These two methods are equivalent up to the leading double logarithm accuracy. More recently, a BK equation with target rapidity being the evolution variable was argued to be superior than that describing projectile rapidity evolution, in the sense that the resummed version of the former one has the less scheme dependence [22].

In this paper, we focus on investigating the contributions from the phase space region beyond the aforementioned strong ordering region. We adopt target rapidity evolution point of view in our analysis. In this scenario, the so-called anti-collinear logarithm appears in the full two loop BK kernel [6] is absent at the NLO. Instead, a double logarithm generated from the phase space region of interest becomes a large correction when one of the daughter dipoles is much smaller than the parent dipole. This finding is consistent with what the authors of the paper [22] observed. Moreover, the fact that the double logarithm terms result from the incomplete cancellation between real corrections and virtual corrections leads us to identify it as the Sudakov logarithm. Consequently, these logarithms can be resummed into an exponential following the standard procedure, and give rise to a Sudakov suppressed BK equation. However, quite puzzlingly, the expansion of the Sudakov suppressed non-local BK equation to the first non-trivial order would produce a double logarithm term that is different from the corresponding one appears in the NLO BK equation with the target rapidity being the evolution variable [22]. Though this mismatch must be clarified at some point, we are not able to offer a solution for the moment, but aim at investigating it thoroughly in a future work.

In recent years, the study of Sudakov resummation in the context of small $x$ physics is actually becoming a topical issue. This was first initiated by the authors of refs. [23, 24], in which a joint resummation formalism was developed to compute physical observables in high energy scattering processes involving at least three well separated scales. In order to formulate the joint $k_{t}$ and small $x$ resummation in the conventional TMD factorization framework, the Collins-Soper evolution and the scale dependence of gluon TMD in the small $x$ limit was later investigated in refs. [25-27]. Such an effective TMD factorization in the small $x$ limit has also been applied in phenomenology studies [28-32]. We notice that the other formulations exist in the literatures [33-36].

The papers is organized as follows. In the next section, we first re-derive the LO BK equation from a t-channel calculation. Following a short discussion about kinematically improved BK equation, we identify the phase space region where the large double logarithm can be produced. We then proceed to analyze the dynamical origin of the double logarithm, and argue how to systemically resum them to all orders. The paper is summarized in section 3 . 


\section{Derivation of the double logarithm correction to the BK kernel at two loop order and beyond}

The large logarithm correction to the BK kernel arises at two loop order. In order to fix the notations and set up the baseline for the two loop calculation, we first briefly review the derivation of the leading order BK evolution kernel. To this end, we compute the NLO correction to the dipole matrix element defined below,

$$
\frac{1}{N_{c}}\left\langle\operatorname{Tr}\left[U\left(x_{\perp}\right) U^{\dagger}\left(y_{\perp}\right)\right]\right\rangle
$$

The Wilson line is defined as,

$$
U\left(x_{\perp}\right)=\mathcal{P} \exp \left\{i g_{s} \int_{-\infty}^{+\infty} d x^{-} A^{+}\left(x^{-}, x_{\perp}\right) T^{c}\right\}
$$

where the plus $(+)$ and the minus $(-)$ symbols represent the commonly defined light cone components of four momenta. At the leading order, the dipole amplitude $\left\langle U\left(x_{\perp}\right) U^{\dagger}\left(y_{\perp}\right)\right\rangle$ is independent of rapidity. It is normally determined in the McLerran-Venugopalan model [37-39] as the initial condition. The large logarithm $\ln \frac{1}{x_{g}}$ shows up in the one loop correction to the above matrix element. These logarithm terms at high orders can be absorbed into the dipole amplitude and give rise to the energy dependence of the correlator.

To avoid the interaction between the radiated gluon and color source inside target, our calculation is performed in the light cone gauge $\left(A^{-}=0\right)$, in which gluon propagator reads,

$$
\left(-g^{\mu \nu}+\frac{P^{\mu} k^{\nu}+P^{\nu} k^{\mu}}{k \cdot P-i \epsilon}\right) \frac{i}{k^{2}+i \epsilon}
$$

where the prescription $\frac{1}{k \cdot P-i \epsilon}$ for regulating the light cone divergence is proven to be the most convenient choice for our calculation.

The real contribution to the BK kernel at the leading order comes from graphs shown in figures $1 \mathrm{a}-1 \mathrm{~d}$. In momentum space, the amplitude of figure 1a is given by,

$$
\mathcal{M}_{1 a}=-i g_{s} \frac{2 k_{\perp} \cdot \epsilon_{\perp}^{*}}{k_{\perp}^{2}} t^{a}\left[U\left(p_{g \perp}\right)-(2 \pi)^{2} \delta^{2}\left(p_{g \perp}\right)\right]
$$

The amplitude of diagrams figure $1 \mathrm{~b}$, figure $1 \mathrm{c}$ and figure $1 \mathrm{~d}$ read,

$$
\begin{aligned}
\mathcal{M}_{1 b}+\mathcal{M}_{1 c}+\mathcal{M}_{1 d}= & -i g_{s} \int \frac{d^{2} p_{g 1 \perp}}{(2 \pi)^{2}} \frac{2\left(k_{\perp}-p_{g 1 \perp}\right) \cdot \epsilon_{\perp}^{*}}{\left(k_{\perp}-p_{g 1 \perp}\right)^{2}} \\
& \times\left[U\left(p_{g \perp}-p_{g 1 \perp}\right) t^{b} \tilde{U}_{a b}\left(p_{g 1 \perp}\right)-(2 \pi)^{4} t^{a} \delta^{2}\left(p_{g 1 \perp}\right) \delta^{2}\left(p_{g \perp}-p_{g 1 \perp}\right)\right]
\end{aligned}
$$

where $\tilde{U}\left(p_{g 1 \perp}\right)$ is the Fourier transform of the path ordered Wilson lines in the adjoint representation. After taking the Fourier transform, the squared summation of the amplitudes figures $1 \mathrm{a}-1 \mathrm{~d}$ in the coordinator space is expressed as,

$$
\frac{g_{s}^{2}}{2 \pi^{2}} \frac{\left(x_{\perp}-z_{\perp}\right) \cdot\left(y_{\perp}-z_{\perp}\right)}{\left(x_{\perp}-z_{\perp}\right)^{2}\left(y_{\perp}-z_{\perp}\right)^{2}}\left\{2 \operatorname{Tr}\left[U^{\dagger}\left(y_{\perp}\right) t^{a} t^{a} U\left(x_{\perp}\right)\right]-2 \operatorname{Tr}\left[U^{\dagger}\left(y_{\perp}\right) U\left(z_{\perp}\right) t^{a} U^{\dagger}\left(z_{\perp}\right) U\left(x_{\perp}\right) t^{a}\right]\right\} .
$$




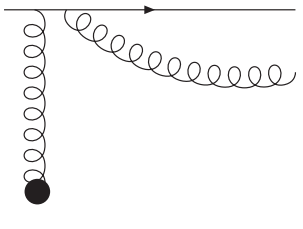

(a)

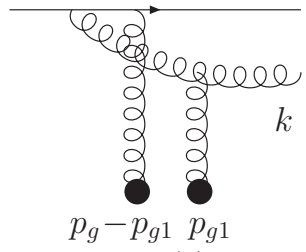

(b)

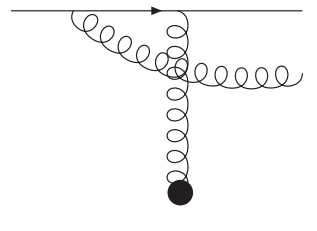

(c)

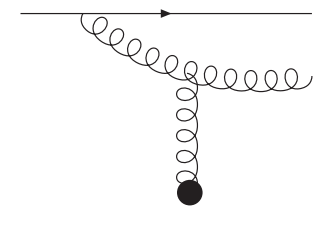

(d)

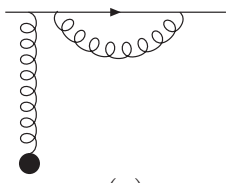

(e)

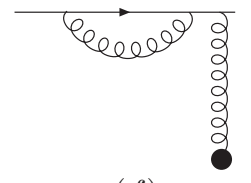

(f)

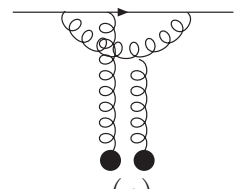

(g)

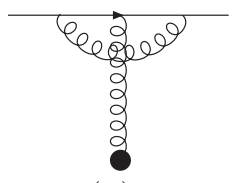

(h)

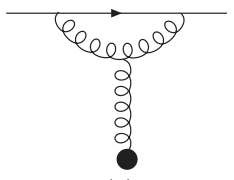

(i)

Figure 1. Real and virtual diagrams which contribute to the BK kernel at the leading order. The black dot stands for multiple gluon re-scattering. The straight solid line represents the Wilson line in the fundamental representation.

To arrive at the above expression, we have used the formula $U^{\dagger}\left(z_{\perp}\right) t^{a} U\left(z_{\perp}\right)=t^{b} \tilde{U}_{a b}\left(z_{\perp}\right)$. By further employing the Fierz identity,

$$
\begin{aligned}
& \operatorname{Tr}\left[U^{\dagger}\left(y_{\perp}\right) U\left(z_{\perp}\right) t^{a} U^{\dagger}\left(z_{\perp}\right) U\left(x_{\perp}\right) t^{a}\right] \\
& =\frac{1}{2} \operatorname{Tr}\left[U^{\dagger}\left(y_{\perp}\right) U\left(z_{\perp}\right)\right] \operatorname{Tr}\left[U^{\dagger}\left(z_{\perp}\right) U\left(x_{\perp}\right)\right]-\frac{1}{2 N_{c}} \operatorname{Tr}\left[U^{\dagger}\left(y_{\perp}\right) U\left(x_{\perp}\right)\right]
\end{aligned}
$$

the real contribution to the BK kernel can be cast into a familiar form,

$$
\frac{g_{s}^{2}}{2 \pi^{2}} \frac{\left(x_{\perp}-z_{\perp}\right) \cdot\left(y_{\perp}-z_{\perp}\right)}{\left(x_{\perp}-z_{\perp}\right)^{2}\left(y_{\perp}-z_{\perp}\right)^{2}}\left\{N_{c} \operatorname{Tr}\left[U^{\dagger}\left(y_{\perp}\right) U\left(x_{\perp}\right)\right]-\operatorname{Tr}\left[U^{\dagger}\left(y_{\perp}\right) U\left(z_{\perp}\right)\right] \operatorname{Tr}\left[U^{\dagger}\left(z_{\perp}\right) U\left(x_{\perp}\right)\right]\right\} .
$$

Similarly, virtual graphs illustrated in figures $1 \mathrm{e}-1 \mathrm{i}$ can be readily computed. Combining the real contribution and the virtual contribution and carrying out phase space integration, one ends up with,

$$
\begin{aligned}
\left\langle\operatorname{Tr}\left[U\left(x_{\perp}\right) U^{\dagger}\left(y_{\perp}\right)\right]\right\rangle= & \left.\left\langle\operatorname{Tr}\left[U\left(x_{\perp}\right) U^{\dagger}\left(y_{\perp}\right)\right]\right\rangle\right|_{\mathrm{LO}}+\frac{\alpha_{s}}{2 \pi^{2}} \int \frac{d k^{+}}{k^{+}} \int d^{2} z_{\perp} \frac{\left(x_{\perp}-y_{\perp}\right)^{2}}{\left(x_{\perp}-z_{\perp}\right)^{2}\left(y_{\perp}-z_{\perp}\right)^{2}} \\
& \times\left\{\operatorname{Tr}\left[U^{\dagger}\left(y_{\perp}\right) U\left(z_{\perp}\right)\right] \operatorname{Tr}\left[U^{\dagger}\left(z_{\perp}\right) U\left(x_{\perp}\right)\right]-N_{c} \operatorname{Tr}\left[U^{\dagger}\left(y_{\perp}\right) U\left(x_{\perp}\right)\right]\right\}
\end{aligned}
$$

which matches the first step iteration of the LO BK equation. There are various ways to regulate the light cone divergence for $k^{+}$integration in the above equation. As long as the calculation is performed in the leading high energy logarithm accuracy, all regularization schemes are equivalent. However, they start to deviate from each other by some subleading logarithms at high order. For the current purpose, we do not need to specify the regularization scheme as the double logarithm under investigation has different dynamical origin. 


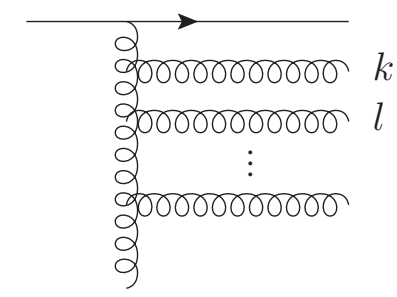

Figure 2. Strong rapidity ordering in successive gluon radiations.

\subsection{Non-local BK equation and kinematical constraints}

When deriving a small $x$ evolution equations like BFKL, BK or B-JIMWLK, it is crucial to impose a strong minus momenta ordering $\bar{P}^{-} \gg k^{-} \gg l^{-} \ldots$ from a projectile evolution point of view as shown in figure 2 , while a strong plus momenta ordering $k^{+} \ll l^{+} \ldots \ll P^{+}$ is required if the problem is formulated as the evolution of a dense target. These two kinematical constraints are compatible with each other so long as the transverse momenta carried by the successive radiated gluons are of the same order $k_{\perp}^{2} \sim l_{\perp}^{2}$. However, this condition can not be always met because transverse momentum is integrated over the whole phase space region in the kernel of the high-energy evolution equations. Instead, it has recently been recognized [18] that it is necessary to have the strong plus momenta ordering and minus momenta ordering simultaneously in order to generate large high energy logarithm from each gluon emission.

In the present paper, we study the NLO BK evolution in $\ln \frac{1}{k^{+}}$, namely the evolution of dense target, for which case the plus momenta are properly ordered by construction. One then should add a theta function in the kernel of the BFKL (or BK) equation in order to impose the $k^{-}$ordering not already guarantied by the choice of evolution variable. The various ways of enforcing such kinematical constraint have been proposed in the literatures [17-22]. These kinematically improved evolution equations effectively resum some large high order corrections to the BFKL/BK kernel to all orders. And in general, a kinematically improved BK equation leads to a more stable small $x$ evolution in comparison with the use of the NLO BK kernel without resumming large transverse logarithms.

However, there exists a phase space region satisfying both conditions $k^{+} \gg l^{+}$and $k^{-} \gg l^{-}$in which QCD dynamics is not yet explored. If transverse momenta carried by the successive emitted gluons are of the same order, this phase space region basically shrinks to zero. When $k_{\perp}^{2} \gg l_{\perp}^{2}$, the corresponding phase region is sufficiently large to potentially induce large logarithm contributions at NLO. This can be best seen from the following phase space integration,

$$
\int \frac{d^{2} l_{\perp}}{l_{\perp}^{2}} \int_{\frac{l_{\perp}^{2}}{k_{\perp}^{2}} k^{+}}^{k^{+}} \frac{d l^{+}}{l^{+}}=\int \frac{d^{2} l_{\perp}}{l_{\perp}^{2}} \ln \frac{k_{\perp}^{2}}{l_{\perp}^{2}}
$$

where the lower integration limit for $l^{+}$integration is determined according to the constraint $k^{-} \gg l^{-}$. The denominators $\frac{1}{l^{+}} \frac{1}{l^{2}}$ in the above formula represent a typical structure arises from a NLO real correction with a soft gluon emitting from an external line. The 

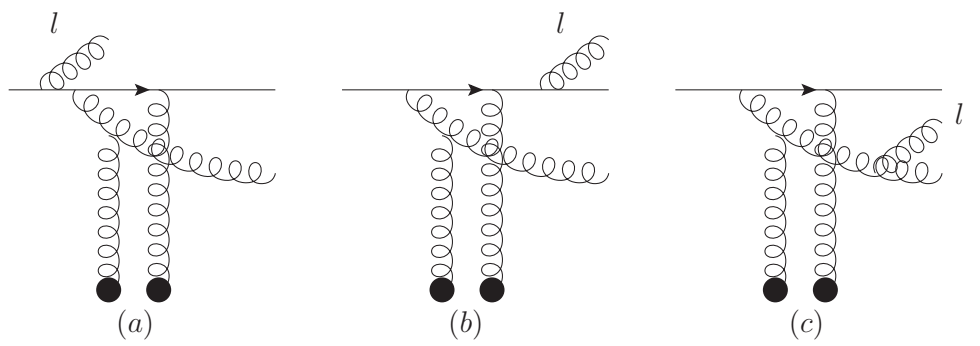

Figure 3. Soft gluon $(l)$ radiation diagrams. Diagrams with the soft gluon radiated from the incoming gluon leg do not produce large Sudakov logarithm.

infrared divergence from the real correction will be canceled out when combining with virtual corrections as explained later. But such cancellation is not complete in the sense that we are left with a double logarithm term. Therefore, on top of the non-local BK equation which already takes care part of large logarithm contributions, one still need to resum these Sudakov type logarithm terms. And once these large logarithms are identified as the typical Sudakov ones, they can be resummed into an exponential by the standard method.

In the following analysis, we focus on the NLO contributions from the phase space region $k^{+} \gg l^{+}$and $k^{-} \gg l^{-}$as the contributions from kinematical regions outside this slice of phase space has been summarized into non-local BK equation. We start presenting the detailed NLO calculations by writing down the amplitudes of graphs illustrated in figure 3. Since all four components of $l$ are much smaller than any scales in the problem under consideration, the soft gluon only can be emitted from external lines. The insertion of the soft gluon to an internal propagator would lead to a power suppressed contribution. Moreover, it is well known that in a light cone gauge calculation, the diagrams with a soft gluon emitting from the incoming gluon lines do not produce any Sudakov type logarithm. Some sample soft gluon radiation diagrams which could potentially generate the large double logarithm are shown in figure 3. Applying the soft gluon approximation, the dominant contributions of the real correction are given by

$$
\begin{aligned}
& \text { Figure 3a } \propto-i g_{s} \frac{2 n^{\mu}}{2 l \cdot n} e^{-i x_{\perp} \cdot l_{\perp}} \mathcal{M}_{\mathrm{LO}}^{c} t^{a} \\
& \text { Figure 3b } \propto i g_{s} \frac{2 n^{\mu}}{2 l \cdot n} e^{-i x_{\perp} \cdot l_{\perp}} t^{a} \mathcal{M}_{\mathrm{LO}}^{c} \\
& \text { Figure 3c } \propto-g_{s} \frac{2 k^{\mu}}{2 l \cdot k} e^{-i z_{\perp} \cdot l_{\perp}} \mathcal{M}_{\mathrm{LO}}^{b} f^{a b c}
\end{aligned}
$$

where the leading order amplitude is denoted as $\mathcal{M}_{\mathrm{LO}}^{c}$ with $c$ being the color index of the gluon $k$. For the conjugate diagrams, one has,

$$
\begin{aligned}
& i g_{s} \frac{2 n^{\mu}}{2 l \cdot n} e^{i y_{\perp} \cdot l_{\perp}} t^{a} \mathcal{M}_{\mathrm{LO}}^{* c}, \\
& -i g_{s} \frac{2 n^{\mu}}{2 l \cdot n} e^{i y_{\perp} \cdot l_{\perp}} \mathcal{M}_{\mathrm{LO}}^{* c} t^{a}, \\
& -g_{s} \frac{2 k^{\mu}}{2 l \cdot k} e^{i z_{\perp} \cdot l_{\perp}} \mathcal{M}_{\mathrm{LO}}^{* b} f^{a b c} .
\end{aligned}
$$




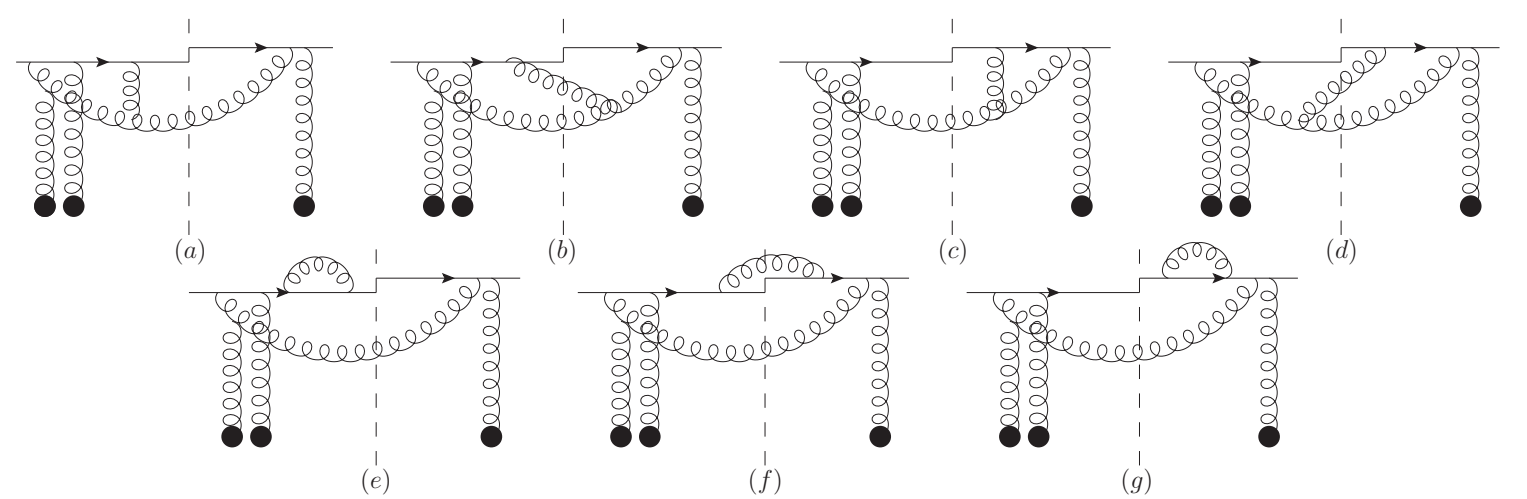

Figure 4. Sample diagrams contributing to the BK kernel at two loop order. The contributions from the figures $4 \mathrm{a}+4 \mathrm{~b}$ are completely canceled out up to the double leading logarithm accuracy. The same cancellation occurs between the figure $4 \mathrm{c}$ and the figure $4 \mathrm{~d}$. In contrast, the double leading logarithm NLO correction to the real part of the LO BK kernel arise from the incomplete cancellation among figure $4 \mathrm{e}$, figure $4 \mathrm{f}$ and figure $4 \mathrm{~g}$.

The virtual graphs with a soft gluon emission/absorbtion can be readily computed in the same approximation as well. Using these derived amplitudes for soft gluon radiations as the basic calculation ingredients, we are ready to extract the double leading logarithm contributions from the NLO correction to the BK kernel.

\subsection{The complete and incomplete cancellations between real graphs and vir- tual graphs}

It is well known that the Sudakov type double logarithm results from the mismatch between soft gluon radiation contributions in real corrections and virtual corrections. In this subsection, we are going to demonstrate that at NLO, such incomplete cancellation indeed occurs in the phase space region $k^{+} \gg l^{+}$and $k^{-} \gg l^{-}$for the diagrams with a soft gluon attaching to the Wilson line. In contrast, if the soft gluon $(l)$ is radiated from the hard gluon $(k)$ line, there is a complete cancellation up to the accuracy of interest between real diagrams and virtual diagrams as illustrated in figures $4 \mathrm{a}-4 \mathrm{~d}$

We first use the figure $4 \mathrm{a}$ and figure $4 \mathrm{~b}$ as an example to show how the leading double logarithm contributions are canceled out. This is essentially due to the fact that the virtual contribution figure $4 \mathrm{a}$ and real contribution figure $4 \mathrm{~b}$ share a common phase factor $e^{-i l_{\perp} \cdot\left(x_{\perp}-z_{\perp}\right)}$. We start with the calculation of the figure 4 a. We first carry out $l^{-}$integration with the residue theorem by picking up the contribution from the pole $1 /\left(l^{2}+i \epsilon\right)$. After having done so, the soft gluon momentum $(l)$ is effectively put on shell. Applying the soft gluon approximation, the virtual correction from figure 4a reads,

Figure $4 \mathrm{a} \propto \int \frac{d k^{+}}{k^{+}} \int d^{2} k_{\perp} \int_{0}^{\left|k_{\perp}\right|} d^{2} l_{\perp} \int_{\frac{l_{\perp}^{2}}{k_{\perp}^{2}}}^{k^{+}} \frac{d l^{+}}{l^{+}} \int d^{2} k_{\perp}^{\prime} d^{2} z_{\perp} \int d^{2} p_{g \perp} d^{2} p_{g \perp}^{\prime} d^{2} p_{g 1 \perp}$

$$
\begin{aligned}
& \times e^{-i\left(z_{\perp}-x_{\perp}\right) \cdot k_{\perp}^{\prime}} e^{i\left(z_{\perp}-y_{\perp}\right) \cdot k_{\perp}} e^{i\left(x_{\perp}-y_{\perp}\right) \cdot l_{\perp}} e^{-i x_{\perp} \cdot p_{g \perp}+i y_{\perp} \cdot p_{g \perp}^{\prime}} \frac{2\left[\left(k_{\perp}+l_{\perp}\right) \cdot\left(k_{\perp}^{\prime}-p_{g 1 \perp}\right)\right]}{\left(k_{\perp}+l_{\perp}\right)^{2}\left(k_{\perp}^{\prime}-p_{g 1 \perp}\right)^{2}} \\
& \times \frac{\left[n \cdot \epsilon_{l}^{*}\right]\left[k \cdot \epsilon_{l}\right]}{[l \cdot n][-k \cdot l]} \operatorname{Tr}\left[U^{\dagger}\left(p_{g \perp}^{\prime}\right) t^{a} t^{b} U\left(p_{g \perp}-p_{g 1 \perp}\right) t^{c}\right] f^{b a d} \tilde{U}\left(p_{g 1 \perp}\right)_{d c}
\end{aligned}
$$


where we shift momentum $k \rightarrow k+l$. The upper limit for $l_{\perp}$ integration is chosen to be $\left|k_{\perp}\right|$. This kinematical constraint is justified because the soft gluon approximation is valid only if $\left|l_{\perp}\right| \ll\left|k_{\perp}\right|$. Moreover, after subtracting the $(\mathrm{LO})^{2}$ contribution which is supposed to be absorbed into non-local BK equation, the upper limit and lower limit for $l^{+}$ integration is determined accordingly. By changing the integration variables $k-p_{g 1} \rightarrow k^{\prime \prime}$ and $k+l \rightarrow k^{\prime \prime \prime}$, the integration over $p_{g \perp}, p_{g \perp}^{\prime}, p_{g 1 \perp}$ can be carried out straightforwardly. After these manipulations, one obtains,

Figure $4 \mathrm{a} \propto \int \frac{d k^{+}}{k^{+}} \int d^{2} k_{\perp} \int_{0}^{\left|k_{\perp}\right|} d^{2} l_{\perp} \int_{\frac{l_{\perp}^{2}}{k_{\perp}^{2}} k^{+}}^{k^{+}} \frac{d l^{+}}{l^{+}} \int d^{2} k_{\perp}^{\prime} d^{2} z_{\perp} e^{-i\left(z_{\perp}-x_{\perp}\right) \cdot k_{\perp}^{\prime \prime}} e^{i\left(z_{\perp}-y_{\perp}\right) \cdot k_{\perp}} e^{i\left(x_{\perp}-z_{\perp}\right) \cdot l_{\perp}}$

$$
\times \frac{2\left[k_{\perp} \cdot k_{\perp}^{\prime \prime}\right]}{k_{\perp}^{2} k_{\perp}^{\prime \prime 2}} \frac{\left[n \cdot \epsilon_{l}^{*}\right]\left[k \cdot \epsilon_{l}\right]}{[l \cdot n][-k \cdot l]} \operatorname{Tr}\left[U^{\dagger}\left(y_{\perp}\right) t^{a} t^{b} U\left(x_{\perp}\right) t^{c}\right] f^{b a d} \tilde{U}\left(z_{\perp}\right)_{d c}
$$

where $k^{\prime \prime \prime}$ has been renamed as $k$.

Similarly, the contribution from figure $4 \mathrm{~b}$ is given by,

Figure $4 \mathrm{~b} \propto \int \frac{d k^{+}}{k^{+}} \int d^{2} k_{\perp} \int_{0}^{\left|k_{\perp}\right|} d^{2} l_{\perp} \int_{\frac{l_{\perp}^{2}}{k_{\perp}^{2}} k^{+}}^{k^{+}} \frac{d l^{+}}{l^{+}} \int d^{2} k_{\perp}^{\prime} d^{2} z_{\perp} \int d^{2} p_{g \perp} d^{2} p_{g \perp}^{\prime} d^{2} p_{g 1 \perp}$

$$
\begin{aligned}
& \times e^{-i\left(z_{\perp}-x_{\perp}\right) \cdot k_{\perp}^{\prime}} e^{i\left(z_{\perp}-y_{\perp}\right) \cdot k_{\perp}} e^{i\left(x_{\perp}-y_{\perp}\right) \cdot l_{\perp}} e^{-i x_{\perp} \cdot p_{g \perp}+i y_{\perp} \cdot p_{g \perp}^{\prime}} \frac{2\left[k_{\perp} \cdot\left(k_{\perp}^{\prime}-p_{g 1 \perp}\right)\right]}{\left(\left(k_{\perp}+l_{\perp}\right)^{2}+2 k \cdot l\right)\left(k_{\perp}^{\prime}-p_{g 1 \perp}\right)^{2}} \\
& \frac{\left[n \cdot \epsilon_{l}^{*}\right]\left[k \cdot \epsilon_{l}\right]}{[l \cdot n][k \cdot l]} \operatorname{Tr}\left[U^{\dagger}\left(p_{g \perp}^{\prime}\right) t^{a} t^{b} U\left(p_{g \perp}-p_{g 1 \perp}\right) t^{c}\right] f^{b a d} \tilde{U}\left(p_{g 1 \perp}\right)_{d c} .
\end{aligned}
$$

One notices that the main contribution to the above integration is from the phase space region where $k \cdot l \rightarrow 0$. We thus can neglect $k \cdot l$ term in the denominator $1 /\left(\left(k_{\perp}+l_{\perp}\right)^{2}+\right.$ $2 k \cdot l)$. By applying the same trick, namely, changing integration variables $k+l \rightarrow k^{\prime \prime \prime}$ and $k^{\prime}-p_{g 1} \rightarrow k^{\prime \prime}$, we can readily integrate out $p_{g \perp}, p_{g \perp}^{\prime}, p_{g 1 \perp}$,

Figure $4 \mathrm{~b} \propto \int \frac{d k^{+}}{k^{+}} \int d^{2} k_{\perp} \int_{0}^{\left|k_{\perp}\right|} d^{2} l_{\perp} \int_{\frac{l_{\perp}^{2}}{k_{\perp}^{2}} k^{+}}^{k^{+}} \frac{d l^{+}}{l^{+}} \int d^{2} k_{\perp}^{\prime} d^{2} z_{\perp} e^{-i\left(z_{\perp}-x_{\perp}\right) \cdot k_{\perp}^{\prime \prime}} e^{i\left(z_{\perp}-y_{\perp}\right) \cdot k_{\perp}} e^{i\left(x_{\perp}-z_{\perp}\right) \cdot l_{\perp}}$

$$
\times \frac{2\left[k_{\perp} \cdot k_{\perp}^{\prime \prime}\right]}{k_{\perp}^{2} k_{\perp}^{\prime \prime 2}} \frac{\left[n \cdot \epsilon_{l}^{*}\right]\left[k \cdot \epsilon_{l}\right]}{[l \cdot n][k \cdot l]} \operatorname{Tr}\left[U^{\dagger}\left(y_{\perp}\right) t^{a} t^{b} U\left(x_{\perp}\right) t^{c}\right] f^{b a d} \tilde{U}\left(z_{\perp}\right)_{d c}
$$

where $k_{\perp}^{\prime \prime \prime}$ has been renamed as $k_{\perp}$. Here some terms suppressed by the power of $l_{\perp}^{2} / k_{\perp}^{2}$ have been ignored. At this step, it is clear to see that contributions from figure 4a and figure $4 \mathrm{~b}$ are canceled out in the soft gluon approximation, and thus do not produce double logarithm. The same analysis also can be applied to other real/virtual diagrams with soft gluon attaching to hard gluon line, for instance figure $4 \mathrm{c}$ and figure $4 \mathrm{~d}$. In the following, we focus on investigating the diagrams with soft gluon emitted from the Wilson lines.

We proceed to compute the double leading logarithm contributions from the diagrams figure $4 \mathrm{e}$, figure $4 \mathrm{f}$ and figure $4 \mathrm{~g}$. The overall extra phase factor associated with real 
diagram figure $4 \mathrm{f}$ is $e^{i l_{\perp} \cdot\left(x_{\perp}-y_{\perp}\right)}$, while no non-trivial phase factor is yielded from the virtual contributions figure $4 \mathrm{e}$ and figure $4 \mathrm{~g}$. Summing up contributions from figure $4 \mathrm{e}-4 \mathrm{~g}$, one obtains,

$$
\begin{aligned}
\text { Figure } 4 \mathrm{e}+4 \mathrm{f}+4 \mathrm{~g}= & g_{s}^{4} \int d^{2} z_{\perp} \int \frac{d k^{+}}{2 \pi k^{+}} \int \frac{d^{2} k_{\perp}}{(2 \pi)^{2}} \frac{d^{2} k_{\perp}^{\prime}}{(2 \pi)^{2}} e^{-i\left(z_{\perp}-x_{\perp}\right) \cdot k_{\perp}^{\prime}} e^{i\left(z_{\perp}-y_{\perp}\right) \cdot k_{\perp}} \\
& \times \int_{0}^{k_{\perp} \mid} \frac{d^{2} l_{\perp}}{(2 \pi)^{2}} \frac{2 C_{F}}{l_{\perp}^{2}}\left\{e^{i\left(x_{\perp}-y_{\perp}\right) \cdot l_{\perp}}-1\right\} \int_{\frac{l_{\perp}^{2}}{k_{\perp}^{2}} k^{+}}^{k^{+}} \frac{d l^{+}}{2 \pi l^{+}} \\
& \times \frac{4\left(k_{\perp} \cdot \epsilon_{k \perp}\right)\left(k_{\perp}^{\prime} \cdot \epsilon_{k \perp}^{*}\right)}{k_{\perp}^{2} k_{\perp}^{\prime 2}} \operatorname{Tr}\left[U^{\dagger}\left(y_{\perp}\right) t^{a} U\left(x_{\perp}\right) t^{b}\right] \tilde{U}\left(z_{\perp}\right)_{a b}
\end{aligned}
$$

where once again, we only take into account the NLO corrections in the phase space region $k^{+} \gg l^{+}$and $k^{-} \gg l^{-}$, beyond which all leading logarithms from the NLO corrections can be organized into non-local BK equation. The next step is to carry out $l^{+}$integration. This subsequently yields a $l_{\perp}$ integration,

$$
\int_{0}^{\left|k_{\perp}\right|} \frac{d^{2} l_{\perp}}{(2 \pi)^{2}} \frac{1}{l_{\perp}^{2}} \ln \frac{k_{\perp}^{2}}{l_{\perp}^{2}}\left\{e^{i\left(x_{\perp}-y_{\perp}\right) \cdot l_{\perp}}-1\right\}
$$

which can produce a large double logarithm $\ln ^{2}\left[k_{\perp}^{2}\left(x_{\perp}-y_{\perp}\right)^{2}\right]$ so long as $k_{\perp}^{2} \gg 1 /\left(x_{\perp}-\right.$ $\left.y_{\perp}\right)^{2}$. After integrating out $k_{\perp}$ and $l_{\perp}$ with the help of the following integration formula,

$$
\begin{aligned}
& \int \frac{d^{2} k_{\perp}}{(2 \pi)^{2}} \frac{k_{\perp} \cdot \epsilon_{k \perp}}{k_{\perp}^{2}} e^{i\left(z_{\perp}-y_{\perp}\right) \cdot k_{\perp}} \int_{0}^{\left|k_{\perp}\right|} \frac{d^{2} l_{\perp}}{(2 \pi)^{2}} \frac{1}{l_{\perp}^{2}} \ln \frac{k_{\perp}^{2}}{l_{\perp}^{2}}\left\{e^{i\left(x_{\perp}-y_{\perp}\right) \cdot l_{\perp}}-1\right\} \\
= & \frac{-1}{(2 \pi)^{2}} \frac{1}{4} \frac{i\left(z_{\perp}-y_{\perp}\right) \cdot \epsilon_{k}}{\left(z_{\perp}-y_{\perp}\right)^{2}} \ln ^{2} \frac{\left(z_{\perp}-y_{\perp}\right)^{2}}{\left(x_{\perp}-y_{\perp}\right)^{2}} \quad\left(\left|x_{\perp}-y_{\perp}\right|>\left|z_{\perp}-y_{\perp}\right|\right)
\end{aligned}
$$

the desired transverse double logarithm shows up. It is then straightforward to carry out the rest integrations. When dust settles, the expression is organized into the form,

$$
\begin{aligned}
& \text { Figure } 4 \mathrm{e}+4 \mathrm{f}+4 \mathrm{~g}=-\frac{C_{F}}{2 \pi^{3}} \alpha_{s}^{2} \int \frac{d k^{+}}{k^{+}} \int d^{2} z_{\perp} \ln ^{2} \frac{\left(x_{\perp}-y_{\perp}\right)^{2}}{\left(z_{\perp}-y_{\perp}\right)^{2}} \\
& \times \frac{\left(x_{\perp}-z_{\perp}\right) \cdot\left(y_{\perp}-z_{\perp}\right)}{\left(x_{\perp}-z_{\perp}\right)^{2}\left(y_{\perp}-z_{\perp}\right)^{2}} \operatorname{Tr}\left[U^{\dagger}\left(y_{\perp}\right) t^{a} U\left(x_{\perp}\right) t^{b}\right] \tilde{U}\left(z_{\perp}\right)_{a b}
\end{aligned}
$$

At this point, it becomes evident that the obtained double transverse logarithm has the typical dynamical origin of a Sudakov problem, as stated before.

The calculations of the rest graphs can be carried out in a similar way. Summing up contributions from all diagrams with the soft gluon being radiated from the Wilson lines, one obtains,

$$
\begin{gathered}
g_{s}^{2} \int \frac{d k^{+}}{2 \pi k^{+}} \int d^{2} z_{\perp} \int \frac{d^{2} k_{\perp} d^{2} k_{\perp}^{\prime}}{(2 \pi)^{4}} g_{s}^{2} \int_{0}^{\left|k_{\perp}\right|} \frac{d^{2} l_{\perp}}{(2 \pi)^{2}} \int_{\frac{l_{\perp}^{2}}{k_{\perp}^{2}} k^{+}}^{k^{+}} \frac{d l^{+}}{2 \pi l^{+}} \frac{2}{l_{\perp}^{2}}\left[e^{-i\left(x_{\perp}-y_{\perp}\right) \cdot l_{\perp}}-1\right] \\
\times \operatorname{Tr}\left[\left(t^{a} \mathcal{M}_{\mathrm{sum}}^{* c}-\mathcal{M}_{\mathrm{sum}}^{* c} t^{a}\right)\left(\mathcal{M}_{\mathrm{sum}}^{c} t^{a}-t^{a} \mathcal{M}_{\mathrm{sum}}^{c}\right)\right]
\end{gathered}
$$


where $\mathcal{M}_{\text {sum }}^{c}$ denotes the summation of all leading order amplitudes. Here the color structure can be simplified using the Fierz identity,

$$
\operatorname{Tr}\left[\left(t^{a} \mathcal{M}_{\mathrm{sum}}^{* c}-\mathcal{M}_{\mathrm{sum}}^{* c} t^{a}\right)\left(\mathcal{M}_{\mathrm{sum}}^{c} t^{a}-t^{a} \mathcal{M}_{\mathrm{sum}}^{c}\right)\right]=N_{c} \operatorname{Tr}\left[\mathcal{M}_{\mathrm{sum}}^{* c} \mathcal{M}_{\mathrm{sum}}^{c}\right]-\operatorname{Tr}\left[\mathcal{M}_{\mathrm{sum}}^{* c}\right] \operatorname{Tr}\left[\mathcal{M}_{\mathrm{sum}}^{c}\right]
$$

To proceed further, we ignore the contribution associated with the second color structure $\operatorname{Tr}\left[\mathcal{M}_{\text {sum }}^{* c}\right] \operatorname{Tr}\left[\mathcal{M}_{\text {sum }}^{c}\right]$ which is suppressed in the large $N_{c}$ limit. The next step is to convert the whole expression in momentum space into that in coordinate space by integrating out hard and soft gluon transverse momentum. We eventually end up with,

$$
\begin{aligned}
& \frac{\alpha_{s}}{2 \pi^{2}} \int \frac{d k^{+}}{k^{+}} \int d^{2} z_{\perp} \frac{2\left(x_{\perp}-z_{\perp}\right) \cdot\left(y_{\perp}-z_{\perp}\right)}{\left(x_{\perp}-z_{\perp}\right)^{2}\left(y_{\perp}-z_{\perp}\right)^{2}}\left[-\frac{\alpha_{s} N_{c}}{4 \pi}\left(\ln ^{2} \frac{\left(x_{\perp}-y_{\perp}\right)^{2}}{\left(z_{\perp}-y_{\perp}\right)^{2}}+\ln ^{2} \frac{\left(x_{\perp}-y_{\perp}\right)^{2}}{\left(z_{\perp}-x_{\perp}\right)^{2}}\right)\right] \\
& \times\left\{N_{c} \operatorname{Tr}\left[U^{\dagger}\left(y_{\perp}\right) U\left(x_{\perp}\right)\right]-\operatorname{Tr}\left[U^{\dagger}\left(y_{\perp}\right) U\left(z_{\perp}\right)\right] \operatorname{Tr}\left[U^{\dagger}\left(z_{\perp}\right) U\left(x_{\perp}\right)\right]\right\} .
\end{aligned}
$$

In the limit $\left(x_{\perp}-y_{\perp}\right)^{2} \sim\left(y_{\perp}-z_{\perp}\right)^{2} \gg\left(x_{\perp}-z_{\perp}\right)^{2}$ or $\left(x_{\perp}-y_{\perp}\right)^{2} \sim\left(x_{\perp}-z_{\perp}\right)^{2} \gg\left(y_{\perp}-z_{\perp}\right)^{2}$, the above expression can be cast into a more compact form,

$$
\begin{aligned}
& \frac{\alpha_{s}}{2 \pi^{2}} \int \frac{d k^{+}}{k^{+}} \int d^{2} z_{\perp} \frac{2\left(x_{\perp}-z_{\perp}\right) \cdot\left(y_{\perp}-z_{\perp}\right)}{\left(x_{\perp}-z_{\perp}\right)^{2}\left(y_{\perp}-z_{\perp}\right)^{2}}\left[-\frac{\alpha_{s} N_{c}}{4 \pi} \ln ^{2} \frac{\left(z_{\perp}-x_{\perp}\right)^{2}}{\left(z_{\perp}-y_{\perp}\right)^{2}}\right] \\
& \times\left\{N_{c} \operatorname{Tr}\left[U^{\dagger}\left(y_{\perp}\right) U\left(x_{\perp}\right)\right]-\operatorname{Tr}\left[U^{\dagger}\left(y_{\perp}\right) U\left(z_{\perp}\right)\right] \operatorname{Tr}\left[U^{\dagger}\left(z_{\perp}\right) U\left(x_{\perp}\right)\right]\right\}
\end{aligned}
$$

which is the NLO correction to the real part of the BK kernel in the double leading logarithm approximation. This result again exhibits a typical factorization structure for a Sudakov problem: the BK dynamics is developed via emitting a hard gluon $(k)$, while the effect induced by the soft gluon radiation is factorized into the double logarithm.

Let us now turn to analyze the NLO correction to the virtual part of the leading order BK kernel. For the virtual graph figure 5b, its contribution can be written as,

$$
\int \frac{d^{4} k}{\left[k^{2}+i \epsilon\right]\left[(k+l)^{2}+i \epsilon\right]\left[k^{-}+l^{-}-i \epsilon\right]\left[k^{-}-i \epsilon\right]} H\left(k, l, p_{g \perp}, p_{g \perp}^{\prime}\right)
$$

where $k^{-}$poles are explicitly shown in the above formula. $H\left(k, l, p_{g \perp}, p_{g \perp}^{\prime}\right)$ represents the rest part of the amplitude. One can carry out contour integration on $k^{-}$by picking up contributions from two poles $1 /\left(k^{2}+i \epsilon\right)$ and $1 /\left((k+l)^{2}+i \epsilon\right)$, respectively,

$$
-i 2 \pi \int d^{4} k\left[\delta\left(k^{2}\right)-\delta\left(k^{2}+2 k \cdot l\right)\right] \frac{H\left(k, l, p_{g \perp}, p_{g \perp}^{\prime}\right)}{[2 k \cdot l]\left[k^{-}+l^{-}-i \epsilon\right]\left[k^{-}-i \epsilon\right]} .
$$

Obviously, two poles contributions cancel out in the soft gluon limit. As a result, this diagram does not produce any large double logarithm term. Such cancellation occurs for the graph figure 5a as well. The similar analysis applies to all diagrams with soft gluon attaching to the hard gluon $(k)$ line. We thus reach the same conclusion that the double leading logarithmic enhanced NLO correction to the virtual part of the LO BK kernel can be generated only if soft gluon is radiated from (or absorbed back to) the Wilson lines. For instance, the combination of figure $5 \mathrm{c}$ and figure $5 \mathrm{~d}$ generates a double logarithm term. 


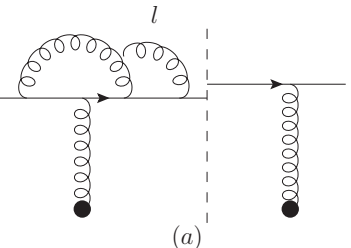

$(a)^{\prime}$

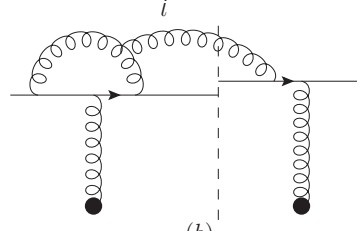

(b)

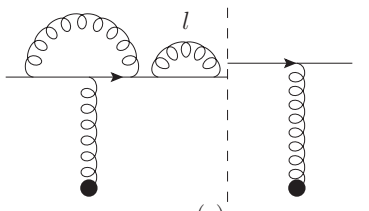

$(c)^{\prime}$

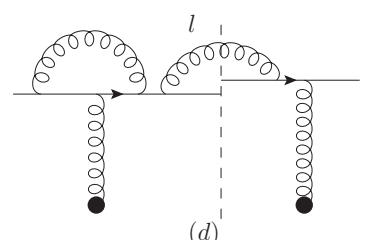

(d)

Figure 5. Sample diagrams contributing to the BK kernel at two loop order. The different pole contributions from figure $5 \mathrm{a}$ and figure $5 \mathrm{~b}$ are completely canceled out within the double leading logarithm accuracy, while the incomplete cancellation between figure $5 \mathrm{c}$ and figure $5 \mathrm{~d}$ gives rise to the partial double leading logarithm NLO correction to the virtual part of the LO BK kernel.

By systematically employing the Eikonal approximation to all such type diagrams, it is straightforward to obtain,

$$
\begin{aligned}
-\frac{\alpha_{s}}{2 \pi^{2}} \int \frac{d k^{+}}{k^{+}} \int & d^{2} z_{\perp}\left\{\frac{1}{\left(x_{\perp}-z_{\perp}\right)^{2}}+\frac{1}{\left(y_{\perp}-z_{\perp}\right)^{2}}\right\}\left[-\frac{\alpha_{s} N_{c}}{4 \pi} \ln ^{2} \frac{\left(z_{\perp}-x_{\perp}\right)^{2}}{\left(z_{\perp}-y_{\perp}\right)^{2}}\right] \\
& \times\left\{N_{c} \operatorname{Tr}\left[U^{\dagger}\left(y_{\perp}\right) U\left(x_{\perp}\right)\right]-\operatorname{Tr}\left[U^{\dagger}\left(y_{\perp}\right) U\left(z_{\perp}\right)\right] \operatorname{Tr}\left[U^{\dagger}\left(z_{\perp}\right) U\left(x_{\perp}\right)\right]\right\}
\end{aligned}
$$

where the double logarithm term is the same as that in eq. (2.24). Combining eq. (2.28) and eq. (2.31) together, one arrives at,

$$
\begin{aligned}
\frac{\partial\left\langle\operatorname{Tr} U^{\dagger}\left(y_{\perp}\right) U\left(x_{\perp}\right)\right\rangle}{\partial \ln \left(1 / x_{g}\right)}= & \frac{\alpha_{s}}{2 \pi^{2}} \int d^{2} z_{\perp} \frac{\left(x_{\perp}-y_{\perp}\right)^{2}}{\left(x_{\perp}-z_{\perp}\right)^{2}\left(y_{\perp}-z_{\perp}\right)^{2}} \\
& \times \frac{1}{2}\left[-\frac{\alpha_{s} N_{c}}{2 \pi} \ln ^{2} \frac{\left(x_{\perp}-y_{\perp}\right)^{2}}{\left(z_{\perp}-x_{\perp}\right)^{2}}-\frac{\alpha_{s} N_{c}}{2 \pi} \ln ^{2} \frac{\left(x_{\perp}-y_{\perp}\right)^{2}}{\left(z_{\perp}-y_{\perp}\right)^{2}}\right] \\
& \times\left\{\left\langle\operatorname{Tr} U^{\dagger}\left(y_{\perp}\right) U\left(z_{\perp}\right)\right\rangle\left\langle\operatorname{Tr} U^{\dagger}\left(z_{\perp}\right) U\left(x_{\perp}\right)\right\rangle-N_{c}\left\langle\operatorname{Tr} U^{\dagger}\left(y_{\perp}\right) U\left(x_{\perp}\right)\right\rangle\right\}
\end{aligned}
$$

which is the final double leading logarithmic enhanced NLO correction to the BK kernel. Note that since our derivation is formulated as dense target evolution, the double leading logarithm term we obtained is different from that appears in the full two loop BK equation describing projectile rapidity evolution [6], but instead coincides with the double logarithm contribution derived in ref. [22] except for a factor $1 / 2$ difference. It is known that the BK equation takes different form at NLO when different evolution variables are used.

\subsection{Multiple soft gluon radiations}

When $\left(x_{\perp}-y_{\perp}\right)^{2} \sim\left(y_{\perp}-z_{\perp}\right)^{2} \gg\left(x_{\perp}-z_{\perp}\right)^{2}$ or $\left(x_{\perp}-y_{\perp}\right)^{2} \sim\left(x_{\perp}-z_{\perp}\right)^{2} \gg\left(y_{\perp}-z_{\perp}\right)^{2}$, the large double logarithm compensates the smallness of the strong coupling constant $\alpha_{s}$ and needs to be summed to all orders to improve the convergence of the perturbative calculation. To this end, one has to take into account multiple soft gluon emissions from the Wilson lines. A schematic graph is shown in figure 6. To simplify our analysis, we take the large $N_{c}$ limit in which the soft gluon can be viewed as being emitted from a 


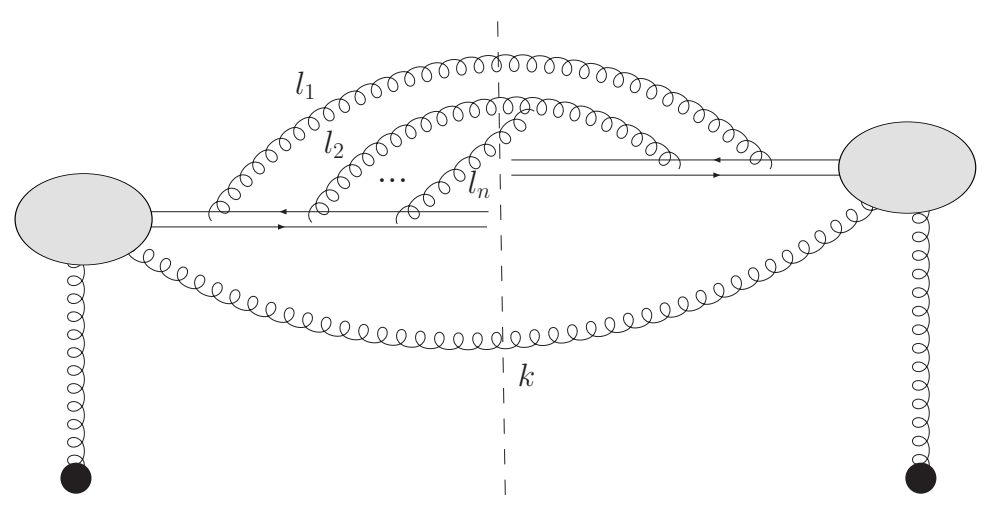

Figure 6. Multiple soft gluon emissions that give rise to the double leading logarithm contributions at high order. The incoming Wilson line can be effectively treated as the outgoing conjugate Wilson line. A pair of the Wilson line and the conjugate Wilson line in the fundamental representation can be viewed as one single Wilson line in the adjoint representation in the large $N_{c}$ limit.

outgoing Wilson line in the adjoint representation. To generate a large logarithm like $\alpha_{s}^{n}\left(\ln \frac{k_{\perp}^{2}\left(x_{\perp}-y_{\perp}\right)^{2}}{c_{0}^{2}}\right)^{2 n}$ at the $\mathrm{n}$-th order, all soft gluon momenta $l_{1}, l_{2} \ldots l_{n}$ must be much smaller than hard gluon momentum $k$. However, one does not have to impose a strong ordering in neither soft gluon transverse momenta nor their longitudinal momenta.

All order resummation of the double leading logarithm terms has been achieved in studying the asymptotic fermion form factor in QED by Sukakov [40] more than half century ago. The algorithm to resum the Sukakov double logarithm in QCD was later developed in refs. [41-43]. Such extension to the non-Abelian gauge theory is highly nontrivial since both ladder diagrams and non-ladder diagrams have to be taken into account. In this work, we resum these large logarithms by following the Collins' factorization approach [44], based on which the soft gluon contribution can be summarized into a form factor $S\left(k_{\perp}^{2},\left(x_{\perp}-y_{\perp}\right)^{2}\right)$ whose operator definition is the same as that for the soft factor often used in the context of TMD factorization. As the hard momentum cut off $-k_{\perp}^{2}$ plays the role of $\zeta_{c}^{2}$ which is a parameter for regularizing the light cone divergence, in the spirit of the factorization [44], we can write down an evolution equation for the form factor in terms of momentum cutoff scale $\mu^{2}$,

$$
\frac{\partial \ln S\left(\mu^{2},\left(x_{\perp}-y_{\perp}\right)^{2}\right)}{\partial \ln \mu^{2}}=-\frac{\alpha_{s} N_{c}}{\pi} \ln \frac{\mu^{2}\left(x_{\perp}-y_{\perp}\right)^{2}}{c_{0}^{2}}
$$

where $c_{0}$ is defined as $c_{0}=2 e^{-\gamma_{E}}$. By setting $S\left(1 /\left(x_{\perp}-y_{\perp}\right)^{2},\left(x_{\perp}-y_{\perp}\right)^{2}\right)=1$, it can be readily deduced from the evolution equation that,

$$
S\left(k_{\perp}^{2},\left(x_{\perp}-y_{\perp}\right)^{2}\right)=\exp \left[-\frac{\alpha_{s} N_{c}}{2 \pi} \ln ^{2} \frac{k_{\perp}^{2}\left(x_{\perp}-y_{\perp}\right)^{2}}{c_{0}^{2}}\right]
$$

where some single logarithm terms are ignored. With the help of the integration formula,

$$
\int d^{2} k_{\perp} \frac{k_{\perp} \cdot \epsilon_{k}^{*}}{k_{\perp}^{2}} e^{-i k_{\perp} \cdot\left(x_{\perp}-z_{\perp}\right)}\left[\ln \frac{k_{\perp}^{2}\left(x_{\perp}-y_{\perp}\right)^{2}}{c_{0}^{2}}\right]^{n}=-i 2 \pi \frac{\left(x_{\perp}-z_{\perp}\right) \cdot \epsilon_{k}^{*}}{\left(x_{\perp}-z_{\perp}\right)^{2}}\left[\ln \frac{\left(x_{\perp}-y_{\perp}\right)^{2}}{\left(x_{\perp}-z_{\perp}\right)^{2}}\right]^{n}+\ldots
$$


we are able to convert the exponential into an expression in the coordinate space,

$$
\exp \left[-\frac{\alpha_{s} N_{c}}{2 \pi} \ln ^{2} \frac{k_{\perp}^{2}\left(x_{\perp}-y_{\perp}\right)^{2}}{c_{0}^{2}}\right] \rightarrow \exp \left[-\frac{\alpha_{s} N_{c}}{2 \pi} \ln ^{2} \frac{\left(x_{\perp}-y_{\perp}\right)^{2}}{\left(z_{\perp}-x_{\perp}\right)^{2}}\right]
$$

or

$$
\exp \left[-\frac{\alpha_{s} N_{c}}{2 \pi} \ln ^{2} \frac{k_{\perp}^{2}\left(x_{\perp}-y_{\perp}\right)^{2}}{c_{0}^{2}}\right] \rightarrow \exp \left[-\frac{\alpha_{s} N_{c}}{2 \pi} \ln ^{2} \frac{\left(x_{\perp}-y_{\perp}\right)^{2}}{\left(z_{\perp}-y_{\perp}\right)^{2}}\right]
$$

Eventually, the Sudakov resummed BK equation takes the form,

$$
\begin{aligned}
\frac{\partial\left\langle\operatorname{Tr} U^{\dagger}\left(y_{\perp}\right) U\left(x_{\perp}\right)\right\rangle}{\partial \ln \left(1 / x_{g}\right)}= & \frac{\alpha_{s}}{2 \pi^{2}} \int d^{2} z_{\perp} \frac{\left(x_{\perp}-y_{\perp}\right)^{2}}{\left(x_{\perp}-z_{\perp}\right)^{2}\left(y_{\perp}-z_{\perp}\right)^{2}} \\
& \times \frac{1}{2}\left\{\exp \left[-\frac{\alpha_{s} N_{c}}{2 \pi} \ln ^{2} \frac{\left(x_{\perp}-y_{\perp}\right)^{2}}{\left(z_{\perp}-y_{\perp}\right)^{2}}\right]+\exp \left[-\frac{\alpha_{s} N_{c}}{2 \pi} \ln ^{2} \frac{\left(x_{\perp}-y_{\perp}\right)^{2}}{\left(z_{\perp}-x_{\perp}\right)^{2}}\right]\right\} \\
& \times\left\{\left\langle\operatorname{Tr} U^{\dagger}\left(y_{\perp}\right) U\left(z_{\perp}\right)\right\rangle\left\langle\operatorname{Tr} U^{\dagger}\left(z_{\perp}\right) U\left(x_{\perp}\right)\right\rangle-N_{c}\left\langle\operatorname{Tr} U^{\dagger}\left(y_{\perp}\right) U\left(x_{\perp}\right)\right\rangle\right\}
\end{aligned}
$$

which is our central result of this work. As mentioned earlier, the expansion of this resummed result to the first non-trivial order matches to the NLO BK kernel for the double transverse logarithm term [22] apart from an additional factor $1 / 2$ difference. Note that the kinematical constraint is not yet explicitly implemented in the above equation. One may expect that a Sudakov suppressed non-local BK equation effectively resums all important sub-leading logarithms to all orders. However, in contrast, it was claimed in ref. [22] that such double transverse logarithm is already encoded in the non-local leading order BK equation. Obviously, this is not consistent with what we found in the present work. But for the time being, we have no clue how to solve this mismatch problem, which undoubtedly deserves further investigation.

\section{Summary}

In this paper, we argue that the large transverse double logarithm arises from the NLO correction to the BK kernel are the typical Sudakov double logarithm which can be resummed to all orders following the standard procedure. To this end, we first identify the main phase region where the double logarithm is generated. As recognized recently, to consistently resum small $x$ logarithm $\ln \frac{1}{x_{g}}$ to all orders, the successive gluon emissions must be simultaneously ordered in longitudinal momenta and in lifetimes;

$$
k^{+} \gg l^{+} \gg \ldots, \quad k^{-} \ll l^{-} \ll \ldots
$$

The implementation of such kinematical constraint results in a non-local BK evolution equation, which effectively resums some sub-leading logarithm terms arise from high order calculations. In this work, we explored the dynamics beyond this strong ordering region, and found that the large double logarithm can be generated in the phase space region where all four components of $l$ is much smaller than that of $k$. It is further shown that the 
resummation of these Sudakov double logarithm can be converted to studying the evolution of soft factor, which has been well formulated in the literatures [44]. Therefore, to resum all important sub-leading logarithms, one has to include a Sukakov factor in the BK kernel in addition to imposing a kinematical constraint. One may expect that such a resummed BK kernel further slows down the small $x$ evolution of dipole. We plan to carry out the detailed numerical study of the Sudakov suppressed non-local BK equation and investigate its phenomenology implications in a future publication.

\section{Acknowledgments}

J. Zhou thanks Feng Yuan for helpful discussions. This work has been supported by the National Science Foundation of China under Grant No. 11675093, and by the Thousand Talents Plan for Young Professionals.

Open Access. This article is distributed under the terms of the Creative Commons Attribution License (CC-BY 4.0), which permits any use, distribution and reproduction in any medium, provided the original author(s) and source are credited.

\section{References}

[1] I. Balitsky, Operator expansion for high-energy scattering, Nucl. Phys. B 463 (1996) 99 [hep-ph/9509348] [INSPIRE].

[2] J. Jalilian-Marian, A. Kovner, A. Leonidov and H. Weigert, The BFKL equation from the Wilson renormalization group, Nucl. Phys. B 504 (1997) 415 [hep-ph/9701284] [INSPIRE].

[3] J. Jalilian-Marian, A. Kovner, A. Leonidov and H. Weigert, The Wilson renormalization group for low x physics: Towards the high density regime, Phys. Rev. D 59 (1998) 014014 [hep-ph/9706377] [INSPIRE].

[4] E. Iancu, A. Leonidov and L.D. McLerran, Nonlinear gluon evolution in the color glass condensate. 1, Nucl. Phys. A 692 (2001) 583 [hep-ph/0011241] [INSPIRE].

[5] Y.V. Kovchegov, Small-x $F_{2}$ structure function of a nucleus including multiple Pomeron exchanges, Phys. Rev. D 60 (1999) 034008 [hep-ph/9901281] [INSPIRE].

[6] I. Balitsky and G.A. Chirilli, Next-to-leading order evolution of color dipoles, Phys. Rev. D 77 (2008) 014019 [arXiv:0710.4330] [INSPIRE].

[7] I. Balitsky and G.A. Chirilli, Rapidity evolution of Wilson lines at the next-to-leading order, Phys. Rev. D 88 (2013) 111501 [arXiv:1309.7644] [InSPIRE].

[8] A. Kovner, M. Lublinsky and Y. Mulian, Jalilian-Marian, Iancu, McLerran, Weigert, Leonidov, Kovner evolution at next to leading order, Phys. Rev. D 89 (2014) 061704 [arXiv: 1310.0378] [INSPIRE].

[9] T. Lappi and H. Mäntysaari, Direct numerical solution of the coordinate space Balitsky-Kovchegov equation at next to leading order, Phys. Rev. D 91 (2015) 074016 [arXiv: 1502.02400] [INSPIRE].

[10] E.A. Kuraev, L.N. Lipatov and V.S. Fadin, The Pomeranchuk Singularity in Nonabelian Gauge Theories, Sov. Phys. JETP 45 (1977) 199 [Zh. Eksp. Teor. Fiz. 72 (1977) 377] [INSPIRE]. 
[11] I.I. Balitsky and L.N. Lipatov, The Pomeranchuk Singularity in Quantum Chromodynamics, Sov. J. Nucl. Phys. 28 (1978) 822 [Yad. Fiz. 28 (1978) 1597] [INSPIRE].

[12] G.P. Salam, A Resummation of large subleading corrections at small x, JHEP 07 (1998) 019 [hep-ph/9806482] [INSPIRE].

[13] M. Ciafaloni, D. Colferai and G.P. Salam, Renormalization group improved small x equation, Phys. Rev. D 60 (1999) 114036 [hep-ph/9905566] [INSPIRE].

[14] M. Ciafaloni, D. Colferai, G.P. Salam and A.M. Stasto, Renormalization group improved small $x$ Green's function, Phys. Rev. D 68 (2003) 114003 [hep-ph/0307188] [INSPIRE].

[15] G. Altarelli, R.D. Ball and S. Forte, Perturbatively stable resummed small x evolution kernels, Nucl. Phys. B 742 (2006) 1 [hep-ph/0512237] [INSPIRE].

[16] M. Ciafaloni, D. Colferai, G.P. Salam and A.M. Stasto, A Matrix formulation for small-x singlet evolution, JHEP 08 (2007) 046 [arXiv: 0707.1453] [INSPIRE].

[17] K. Kutak, K. Golec-Biernat, S. Jadach and M. Skrzypek, Nonlinear equation for coherent gluon emission, JHEP 02 (2012) 117 [arXiv:1111.6928] [INSPIRE].

[18] G. Beuf, Improving the kinematics for low-x QCD evolution equations in coordinate space, Phys. Rev. D 89 (2014) 074039 [arXiv:1401.0313] [INSPIRE].

[19] E. Iancu, J.D. Madrigal, A.H. Mueller, G. Soyez and D.N. Triantafyllopoulos, Resumming double logarithms in the QCD evolution of color dipoles, Phys. Lett. B 744 (2015) 293 [arXiv: 1502.05642] [INSPIRE].

[20] E. Iancu, J.D. Madrigal, A.H. Mueller, G. Soyez and D.N. Triantafyllopoulos, Collinearly-improved BK evolution meets the HERA data, Phys. Lett. B 750 (2015) 643 [arXiv: 1507.03651] [INSPIRE].

[21] T. Lappi and H. Mäntysaari, Next-to-leading order Balitsky-Kovchegov equation with resummation, Phys. Rev. D 93 (2016) 094004 [arXiv: 1601.06598] [INSPIRE].

[22] B. Ducloué, E. Iancu, A.H. Mueller, G. Soyez and D.N. Triantafyllopoulos, Non-linear evolution in QCD at high-energy beyond leading order, JHEP 04 (2019) 081 [arXiv: 1902.06637] [INSPIRE].

[23] A.H. Mueller, B.-W. Xiao and F. Yuan, Sudakov Resummation in Small-x Saturation Formalism, Phys. Rev. Lett. 110 (2013) 082301 [arXiv:1210.5792] [INSPIRE].

[24] A.H. Mueller, B.-W. Xiao and F. Yuan, Sudakov double logarithms resummation in hard processes in the small-x saturation formalism, Phys. Rev. D 88 (2013) 114010 [arXiv: 1308.2993] [INSPIRE].

[25] J. Zhou, The evolution of the small x gluon TMD, JHEP 06 (2016) 151 [arXiv:1603.07426] [INSPIRE].

[26] B.-W. Xiao, F. Yuan and J. Zhou, Transverse Momentum Dependent Parton Distributions at Small-x, Nucl. Phys. B 921 (2017) 104 [arXiv:1703.06163] [INSPIRE].

[27] J. Zhou, Scale dependence of the small $x$ transverse momentum dependent gluon distribution, Phys. Rev. D 99 (2019) 054026 [arXiv: 1807.00506] [INSPIRE].

[28] L. Zheng, E.C. Aschenauer, J.H. Lee and B.-W. Xiao, Probing Gluon Saturation through Dihadron Correlations at an Electron-Ion Collider, Phys. Rev. D 89 (2014) 074037 [arXiv: 1403.2413] [INSPIRE]. 
[29] A. van Hameren, P. Kotko, K. Kutak and S. Sapeta, Small-x dynamics in forward-central dijet decorrelations at the LHC, Phys. Lett. B 737 (2014) 335 [arXiv: 1404.6204] [INSPIRE].

[30] A. van Hameren, P. Kotko, K. Kutak and S. Sapeta, Broadening and saturation effects in dijet azimuthal correlations in $p$ - $p$ and $p$-Pb collisions at $\sqrt{s}=5.02$ TeV, Phys. Lett. B 795 (2019) 511 [arXiv: 1903.01361] [INSPIRE].

[31] D. Boer, P.J. Mulders, J. Zhou and Y.-j. Zhou, Suppression of maximal linear gluon polarization in angular asymmetries, JHEP 10 (2017) 196 [arXiv:1702.08195] [INSPIRE].

[32] H. Dong, D.-X. Zheng and J. Zhou, Sea quark Sivers distribution, Phys. Lett. B 788 (2019) 401 [arXiv: 1805. 09479] [INSPIRE].

[33] I. Balitsky and A. Tarasov, Rapidity evolution of gluon TMD from low to moderate $x$, JHEP 10 (2015) 017 [arXiv: 1505. 02151] [INSPIRE].

[34] I. Balitsky and A. Tarasov, Gluon TMD in particle production from low to moderate $x$, JHEP 06 (2016) 164 [arXiv: 1603.06548] [INSPIRE].

[35] I. Balitsky and G.A. Chirilli, Conformal invariance of transverse-momentum dependent parton distributions rapidity evolution, Phys. Rev. D 100 (2019) 051504(R) [arXiv: 1905.09144] [INSPIRE].

[36] S. Marzani, Combining $Q_{T}$ and small-x resummations, Phys. Rev. D 93 (2016) 054047 [arXiv: 1511.06039] [INSPIRE].

[37] L.D. McLerran and R. Venugopalan, Computing quark and gluon distribution functions for very large nuclei, Phys. Rev. D 49 (1994) 2233 [hep-ph/9309289] [INSPIRE].

[38] L.D. McLerran and R. Venugopalan, Gluon distribution functions for very large nuclei at small transverse momentum, Phys. Rev. D 49 (1994) 3352 [hep-ph/9311205] [INSPIRE].

[39] L.D. McLerran and R. Venugopalan, Green's functions in the color field of a large nucleus, Phys. Rev. D 50 (1994) 2225 [hep-ph/9402335] [INSPIRE].

[40] V.V. Sudakov, Vertex parts at very high-energies in quantum electrodynamics, Sov. Phys. JETP 3 (1956) 65 [Zh. Eksp. Teor. Fiz. 30 (1956) 87] [INSPIRE].

[41] J.M. Cornwall and G. Tiktopoulos, Infrared Behavior of Nonabelian Gauge Theories, Phys. Rev. D 13 (1976) 3370 [INSPIRE].

[42] V.V. Belokurov and N.I. Usyukina, On Exponentiation Of The Singlet Quark Form-Factor In The Leading Logarithm Approximation, Phys. Lett. B 94 (1980) 251 [INSPIRE].

[43] A. Sen, Asymptotic Behavior of the Sudakov Form-Factor in QCD, Phys. Rev. D 24 (1981) 3281 [INSPIRE].

[44] J.C. Collins, Foundations of perturbative QCD, Cambridge University Press, Cambridge U.K. (2011). 